\title{
SOLUTION TO A QUESTION ON A FAMILY OF IMPRIMITIVE SYMMETRIC GRAPHS
}

\author{
GUANGJUN XU ${ }^{\bowtie}$ and SANMING ZHOU
}

(Received 28 September 2009)

\begin{abstract}
We answer a recent question posed by Li et al. ['Imprimitive symmetric graphs with cyclic blocks', European J. Combin. 31 (2010), 362-367] regarding a family of imprimitive symmetric graphs.

2000 Mathematics subject classification: primary 05C25; secondary 05E99.

Keywords and phrases: symmetric graph, arc-transitive graph, quotient graph.
\end{abstract}

A graph $\Gamma=(V, E)$ is called $G$-symmetric if $\Gamma$ admits $G$ as a group of automorphisms such that $G$ is transitive on $V$ and on the set of arcs of $\Gamma$, where an $\operatorname{arc}$ is an ordered pair of adjacent vertices. If in addition $\Gamma$ admits a nontrivial $G$-invariant partition, that is, a partition $\mathcal{B}$ of $V$ such that $1<|B|<|V|$ and $B^{g}:=\left\{\alpha^{g}: \alpha \in B\right\} \in \mathcal{B}$ for $B \in \mathcal{B}$ and $g \in G$, then $\Gamma$ is called an imprimitive $G$-symmetric graph. In this case the quotient graph $\Gamma_{\mathcal{B}}$ of $\Gamma$ with respect to $\mathcal{B}$ is defined to have vertex set $\mathcal{B}$ such that $B, C \in \mathcal{B}$ are adjacent if and only if there exists at least one edge of $\Gamma$ between $B$ and $C$. We assume that $\Gamma_{\mathcal{B}}$ contains at least one edge, so that each block of $\mathcal{B}$ is an independent set of $\Gamma$. Denote by $\Gamma(\alpha)$ the neighbourhood of $\alpha \in V$ in $\Gamma$, and define $\Gamma(X)=\bigcup_{\alpha \in X} \Gamma(\alpha)$ for $X \in \mathcal{B}$. For blocks $B, C \in \mathcal{B}$ adjacent in $\Gamma_{\mathcal{B}}$, let $\Gamma[B, C]$ be the bipartite subgraph of $\Gamma$ induced on $(B \cap \Gamma(C)) \cup(C \cap \Gamma(B))$. Then $\Gamma[B, C]$ is independent of the choice of $(B, C)$ up to isomorphism. Define

$$
v:=|B| \quad \text { and } \quad k:=|B \cap \Gamma(C)|
$$

to be the block size of $\mathcal{B}$ and the size of each part of the bipartition of $\Gamma[B, C]$, respectively.

In line with a geometrical approach suggested in [1], various situations may occur for $\Gamma, G, \Gamma_{\mathcal{B}}, \Gamma[B, C]$ and a certain 1-design with point set $B$; see, for example, [1, 3, 5-7]. The case where $k=v-2 \geq 1$ was studied in [2, 4] and a necessary and sufficient condition for $\Gamma_{\mathcal{B}}$ to be $(G, 2)$-arc-transitive was given in [2]. In this case, the multigraph $\Gamma^{B}$ [2] with vertex $B$ and an edge joining the two vertices of $B \backslash \Gamma(C)$ for every $C \in \Gamma_{\mathcal{B}}(B)$ plays an important role in the structure of $\Gamma$ and $\Gamma_{\mathcal{B}}$,

The first author acknowledges support of an MIFRS and an SFS from The University of Melbourne.

(c) 2010 Australian Mathematical Publishing Association Inc. 0004-9727/2010 \$16.00 
where $\Gamma_{\mathcal{B}}(B)$ is the neighbourhood of $B$ in $\Gamma_{\mathcal{B}}$. Since $\Gamma$ is $G$-symmetric, up to isomorphism $\Gamma^{B}$ is independent of the choice of $B$, and the multiplicity of each edge $\{\alpha, \beta\}$ of $\Gamma^{B}$, namely

$$
m:=\left|\left\{C \in \Gamma_{\mathcal{B}}(B): B \backslash \Gamma(C)=\{\alpha, \beta\}\right\}\right|,
$$

is independent of the choice of $\{\alpha, \beta\}$. Denote by $\operatorname{Simple}\left(\Gamma^{B}\right)$ the underlying simple graph of $\Gamma^{B}$ and by $G_{B}$ the setwise stabilizer of $B$ in $G$. It has been proved [2, Theorem 2.1] that Simple $\left(\Gamma^{B}\right)$ is $G_{B}$-vertex-transitive and $G_{B}$-edge-transitive, and either $\Gamma^{B}$ is connected or $v$ is even and $\operatorname{Simple}\left(\Gamma^{B}\right)$ is a perfect matching $(v / 2) \cdot K_{2}$. In the latter case detailed information about $\Gamma$ was obtained in [2, Theorem 1.3] when $\Gamma^{B}$ is simple. In [4], Li et al. proved that, if $\operatorname{Simple}\left(\Gamma^{B}\right)$ is a cycle, then $v$ must be small, namely $v$ is equal to 3 or 4 . Based on this they posed the following question.

QUESTION 1. In the case where $k=v-2$ and $\Gamma^{B}$ is connected, is $v$ bounded by some function of the valency of Simple $\left(\Gamma^{B}\right)$ ?

Define

$$
b:=\operatorname{val}\left(\Gamma_{\mathcal{B}}\right), \quad s:=\operatorname{val}(\Gamma[B, C]), \quad r:=|\{C \in \mathcal{B}: \alpha \in \Gamma(C)\}|
$$

to be respectively the valency of $\Gamma_{\mathcal{B}}$, the valency of $\Gamma[B, C]$, and the number of blocks of $\mathcal{B}$ that contain at least one neighbour of a fixed vertex $\alpha \in V$ in $\Gamma$. Note that $v, k, b, r$ and $s$ all rely on the $G$-invariant partition $\mathcal{B}$.

In this paper we answer Question 1 by proving the following stronger result: there are only two possibilities for $\mathrm{Simple}\left(\Gamma^{B}\right)$ and $v$ can take two values only.

THEOREM 2. Suppose that $\Gamma$ is a G-symmetric graph which admits a nontrivial $G$-invariant partition $\mathcal{B}$ such that $k=v-2 \geq 1, \Gamma_{\mathcal{B}}$ is connected and $\operatorname{Simple}\left(\Gamma^{B}\right)$ is connected with valency $d \geq 2$. Then one of the following occurs.

(a) Simple $\left(\Gamma^{B}\right) \cong K_{v}, v=d+1, b=m(v-1) v / 2$, and $G_{B}^{B}$ is 2-homogeneous.

(b) Simple $\left(\Gamma^{B}\right) \cong K_{v / 2, v / 2}, \quad v=2 d, \quad b=m v^{2} / 4$, and the bipartition of Simple $\left(\Gamma^{B}\right)$ induces a $G$-invariant partition $\mathcal{B}^{*}$ of the vertex set of $\Gamma$ (which is a refinement of $\mathcal{B})$ such that one of the following holds for its parameters:

(i) $v^{*}=k^{*}+1=v / 2, b^{*}=b, r^{*}=r, s^{*}=s$;

(ii) $v^{*}=k^{*}+1=v / 2, b^{*}=2 b, r^{*}=2 r, s^{*}=s / 2$;

(iii) $v^{*}=2 k^{*}+1=v / 2, b^{*}=2 b, r^{*}=r, s^{*}=s$.

Proof. Suppose that $\Gamma, G$ and $\mathcal{B}$ satisfy the conditions in the theorem. Denote $\Omega:=\operatorname{Simple}\left(\Gamma^{B}\right)$. Let $B$ and $C$ be two blocks of $\mathcal{B}$ adjacent in $\Gamma_{\mathcal{B}}$, and let $\{\alpha, \beta\}=B \backslash \Gamma(C)$ be the corresponding edge of $\Omega$. Define

$$
U:=(\Omega(\alpha) \cup \Omega(\beta)) \backslash\{\alpha, \beta\}
$$

to be the neighbourhood of the subset $\{\alpha, \beta\}$ of $B$ in $\Omega$, and set

$$
W:=B \backslash(U \cup\{\alpha, \beta\}) .
$$


Since $\Omega$ has valency $d \geq 2$, we have $U \neq \emptyset$. Since every element of $G_{B C}\left(=\left(G_{B}\right)_{C}\right)$ fixes $\{\alpha, \beta\}$ setwise, it follows that every element of $G_{B C}$ fixes each of $U$ and $W$ setwise. Thus $G_{B C} \leq G_{U} \cap G_{W}$.

Claim 1. $W=\emptyset$, that is, $U=B \backslash\{\alpha, \beta\}$, or every vertex in $B$ is adjacent to at least one of $\alpha$ and $\beta$ in $\Omega$.

Suppose otherwise and let $\delta \in W$. Since $U \neq \emptyset$, we may take a vertex $\gamma \in U$. Since $\delta, \gamma \neq \alpha, \beta$, there exist $\delta_{1}, \gamma_{1} \in C$ adjacent to $\delta, \gamma$ in $\Gamma$, respectively. (It may occur that $\delta_{1}=\gamma_{1}$.) Since $\Gamma$ is $G$-symmetric, there exists $g \in G$ such that $\left(\gamma, \gamma_{1}\right)^{g}=\left(\delta, \delta_{1}\right)$. Since $g$ maps $\gamma \in B$ to $\delta \in B$ and $\gamma_{1} \in C$ to $\delta_{1} \in C$, it fixes $B$ and $C$ setwise. Hence $g \in G_{B C} \leq G_{U} \cap G_{W}$. However, this is a contradiction, because $g$ maps $\gamma \in U$ to $\delta \in W$. Therefore $W=\emptyset$ as claimed.

Since $\Omega$ has valency $d$, by Claim $1, d-1 \leq|U| \leq 2(d-1)$. Since $v=|U|+2$ by Claim 1, it follows that

$$
d+1 \leq v \leq 2 d .
$$

Claim 2. In $\Omega$ any two adjacent vertices have $2 d-v$ common neighbours, and two nonadjacent vertices have the same neighbourhood.

In fact, since $\Omega$ is $G_{B}$-edge-transitive [2, Theorem 2.1], the number $\lambda$ of common neighbours of a pair of adjacent vertices in $\Omega$ is a constant. Consider the neighbourhood $U$ of $\{\alpha, \beta\}$ in $\Omega$, where $\alpha$ and $\beta$ are as above. There are exactly $d-\lambda-1$ vertices in $B$ which are adjacent to $\alpha$ but not $\beta$ ( $\beta$ but not $\alpha$, respectively).

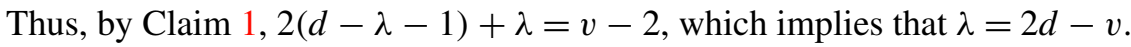

Now let $\sigma$ and $\tau$ be any two nonadjacent vertices of $\Omega$. If $\gamma \in B$ is adjacent to $\sigma$ in $\Omega$, then by applying Claim 1 to the edge $\{\sigma, \gamma\}$, every vertex in $B$ is adjacent to either $\sigma$ or $\gamma$ in $\Omega$. Thus, since $\tau$ is not adjacent to $\sigma$, it must be adjacent to $\gamma$ in $\Omega$ and so $\Omega(\sigma) \subseteq \Omega(\tau)$. Similarly, $\Omega(\tau) \subseteq \Omega(\sigma)$. Hence $\Omega(\sigma)=\Omega(\tau)$ and Claim 2 is proved.

Consider any maximal (with respect to set-theoretic inclusion) independent set $X$ of $\Omega$. By Claim 2 the vertices in $X$ have the same neighbourhood in $\Omega$. Denote this common neighbourhood by $Y$, so that $|Y|=d$. If $B \backslash(X \cup Y) \neq \emptyset$, then by the maximality of $X$, any vertex in $B \backslash(X \cup Y)$ must be adjacent to at least one vertex $\delta \in X$ in $\Omega$, which implies that $\delta$ is adjacent to $d+1$ vertices in $\Omega$. This contradiction shows that $X \cup Y=B$ and consequently $|X|=v-d$. Since this holds for any maximal independent set of $\Omega$ and since $\Omega$ is $G_{B}$-vertex-transitive, we have the following claim.

Claim 3. $v-d$ divides $d$ and $\Omega$ is a complete $t$-partite graph with each part containing $v-d$ vertices, where $t=v /(v-d)$.

Based on this we now prove the following claim.

Claim 4. $\Omega \cong K_{v}$ or $K_{v / 2, v / 2}$; that is, $t=v$ or 2 . 
Suppose to the contrary that $2<t<v$. Denote by $B^{1}, B^{2}, \ldots, B^{t}$ the parts of the $t$-partition of $\Omega$. Similarly, for any $D \in \mathcal{B}$, denote by $D^{1}, D^{2}, \ldots, D^{t}$ the parts of the $t$-partition of Simple $\left(\Gamma^{D}\right)(\cong \Omega)$. Set

$$
\mathcal{B}^{*}:=\left\{D^{1}, D^{2}, \ldots, D^{t}: D \in \mathcal{B}\right\} .
$$

It is straightforward to verify that $\mathcal{B}^{*}$ is a nontrivial $G$-invariant partition of the vertex set of $\Gamma$ and that $\mathcal{B}^{*}$ is a refinement of $\mathcal{B}$. For adjacent $B, C \in \mathcal{B}$ and $\{\alpha, \beta\}=$ $B \backslash \Gamma(C)$ as above, $\alpha$ and $\beta$ belong to different parts of $\Omega$, and so we may assume that $\alpha \in B^{1}$ and $\beta \in B^{2}$ without loss of generality. Since $t<v$, each part of $\Omega$ has size at least two and hence we can take a vertex $\xi \in B^{2} \backslash\{\beta\}$. Since $t>2$, $\Omega$ has at least three parts and so we can take a vertex $\eta \in B^{3}$. Since $B \backslash \Gamma(C)=\{\alpha, \beta\}$ and $\xi, \eta \neq \alpha, \beta$, each of $\xi$ and $\eta$ has at least one neighbour in $C$. Let $\xi$ be adjacent to $\gamma \in C$ and $\eta$ adjacent to $\delta \in C$. Since $\Gamma$ is $G$-symmetric, there exists an element $g \in G$ which maps $(\eta, \delta)$ to $(\xi, \gamma)$. Thus $g \in G_{B C}$. Since $\mathcal{B}^{*}$ is $G$-invariant and $g$ maps $\eta \in B^{3}$ to $\xi \in B^{2}, g$ should map $B^{3}$ to $B^{2}$. Since every vertex in $B^{3}$ has a neighbour in $C$, it follows that every vertex in $B^{2}$ has a neighbour in $C$. However, this is a contradiction since $\beta \in B^{2}$ has no neighbour in $C$. Therefore we have proved Claim 4 .

Obviously, if $\Omega \cong K_{v}$, then $d=v-1, b=m d v / 2=m(v-1) v / 2$, and moreover $G_{B}$ is 2-homogeneous on $B$ since $\Omega$ is $G_{B}$-edge-transitive by [2, Theorem 2.1].

In the case $\Omega \cong K_{v / 2, v / 2}$, we have $d=v / 2, b=m d v / 2=m v^{2} / 4$, and the $G$ invariant partition $\mathcal{B}^{*}$ above becomes $\mathcal{B}^{*}=\left\{D^{1}, D^{2}: D \in \mathcal{B}\right\}$. Obviously, $\mathcal{B}^{*}$ is a nontrivial partition of the vertex set of $\Gamma$ and is a refinement of $\mathcal{B}$. In the case where each of $\Gamma\left(B^{1}\right)$ and $\Gamma\left(B^{2}\right)$ has nonempty intersection with exactly one of $C^{1}$ and $C^{2}$, it is easy to see that $v^{*}=k^{*}+1, b=b^{*}, r=r^{*}$ and $s=s^{*}$, and so case (b)(i) occurs. In the remaining case, each of $\Gamma\left(B^{1}\right)$ and $\Gamma\left(B^{2}\right)$ has nonempty intersection with both $C^{1}$ and $C^{2}$, and hence $b^{*}=2 b$. If further every vertex in $B^{1} \backslash\{\alpha\}$ has neighbours in both $C^{1}$ and $C^{2}$, then $v^{*}=k^{*}+1, r^{*}=2 r$ and $s^{*}=s / 2$, and so case (b)(ii) occurs. If not every vertex in $B^{1} \backslash\{\alpha\}$ has neighbours in both $C^{1}$ and $C^{2}$, then by symmetry the numbers of vertices in $B^{1} \backslash\{\alpha\}$ having neighbours in $C^{1}$ and $C^{2}$ are equal. This implies that

$$
k^{*}=\left(v^{*}-1\right) / 2, \quad r^{*}=b^{*} k^{*} / v^{*}=b(v-2) / v=r \quad \text { and } \quad s^{*}=r s / r^{*}=s,
$$

and hence case (b)(iii) occurs.

Example 2.4 in [2] can serve as an example for case (a) in Theorem 2 when $v=3$. Examples for case (b)(i) when $v=4$ can be obtained from [4, Construction 3.2]: let $M$ be a regular map on a closed surface such that its underlying graph $\Sigma$ has valency four. (A regular map is a 2-cell embedding of a connected (multi)graph on a closed surface such that its automorphism group is regular on incident vertex-edge-face triples.) For each edge $\left\{\sigma, \sigma^{\prime}\right\}$ of $\Sigma$, let $f$ and $f^{\prime}$ denote the faces of $M$ with $\left\{\sigma, \sigma^{\prime}\right\}$ as a common edge. Denote by $f_{\sigma}$ and $f_{\sigma}^{\prime}$ the other two faces of $M$ incident with $\sigma$ and opposite to $f$ and $f^{\prime}$ respectively, and define $f_{\sigma^{\prime}}$ and $f_{\sigma^{\prime}}^{\prime}$ similarly. Let $\Gamma_{1}(M), \Gamma_{2}(M), \Gamma_{3}(M)$ and $\Gamma_{4}(M)$ be the graphs [4] with vertices the incident vertex-face pairs of $M$ and 
adjacency defined as follows (where $\sim$ means adjacency): for each edge $\left\{\sigma, \sigma^{\prime}\right\}$ of $\Sigma,(\sigma, f) \sim\left(\sigma^{\prime}, f\right)$ and $\left(\sigma, f^{\prime}\right) \sim\left(\sigma^{\prime}, f^{\prime}\right)$ in $\Gamma_{1}(M) ;(\sigma, f) \sim\left(\sigma^{\prime}, f^{\prime}\right)$ and $\left(\sigma, f^{\prime}\right) \sim$ $\left(\sigma^{\prime}, f\right)$ in $\Gamma_{2}(M)$; $\left(\sigma, f_{\sigma}\right) \sim\left(\sigma^{\prime}, f_{\sigma^{\prime}}\right)$ and $\left(\sigma, f_{\sigma}^{\prime}\right) \sim\left(\sigma^{\prime}, f_{\sigma^{\prime}}^{\prime}\right)$ in $\Gamma_{3}(M) ;\left(\sigma, f_{\sigma}\right) \sim$ $\left(\sigma^{\prime}, f_{\sigma^{\prime}}^{\prime}\right)$ and $\left(\sigma, f_{\sigma}^{\prime}\right) \sim\left(\sigma^{\prime}, f_{\sigma^{\prime}}\right)$ in $\Gamma_{4}(M)$. These graphs are $G$-symmetric [4, Lemma 3.3] and admit $\mathcal{B}:=\{B(\sigma): \sigma \in V(\Sigma)\}$ as a $G$-invariant partition, where $B(\sigma)=\{(\sigma, f): \sigma$ incident with $f\}$, such that $k=v-2=2, \Gamma_{\mathcal{B}} \cong \Sigma, \Gamma^{B(\sigma)}=K_{2,2}$ and $\Gamma[B(\sigma), B(\tau)]=2 \cdot K_{2}$ for adjacent $B(\sigma), B(\tau) \in \mathcal{B}$. These four graphs fall into case (b)(i) in Theorem 2 and the $G$-invariant partition induced by the bipartition of $\Gamma^{B(\sigma)}$ is $\mathcal{B}^{*}:=\left\{B^{1}(\sigma), B^{2}(\sigma): \sigma \in V(\Sigma)\right\}$, where $B^{1}(\sigma)=\left\{(\sigma, f),\left(\sigma, f_{\sigma}\right)\right\}$ and $B^{2}(\sigma)=\left\{\left(\sigma, f^{\prime}\right),\left(\sigma, f_{\sigma}^{\prime}\right)\right\}$.

\section{References}

[1] A. Gardiner and C. E. Praeger, 'A geometrical approach to imprimitive graphs', Proc. London Math. Soc. (3) 71 (1995), 524-546.

[2] M. A. Iranmanesh, C. E. Praeger and S. Zhou, 'Finite symmetric graphs with two-arc transitive quotients', J. Combin. Theory (Ser. B) 94 (2005), 79-99.

[3] C. H. Li, C. E. Praeger and S. Zhou, 'A class of finite symmetric graphs with 2-arc transitive quotients', Math. Proc. Cambridge Philos. Soc. 129 (2000), 19-34.

[4] C. H. Li, C. E. Praeger and S. Zhou, 'Imprimitive symmetric graphs with cyclic blocks', European J. Combin. 31 (2010), 362-367.

[5] Z. Lu and S. Zhou, 'Finite symmetric graphs with 2-arc transitive quotients (II)', J. Graph Theory 56 (2007), 167-193.

[6] S. Zhou, 'Constructing a class of symmetric graphs', European J. Combin. 23 (2002), 741-760.

[7] S. Zhou, 'Almost covers of 2-arc transitive graphs', Combinatorica 24 (2004), 731-745; [Erratum: 27 (2007), 745-746].

GUANGJUN XU, Department of Mathematics and Statistics, The University of Melbourne, Parkville, Vic 3010, Australia e-mail: gx@ms.unimelb.edu.au

SANMING ZHOU, Department of Mathematics and Statistics, The University of Melbourne, Parkville, Vic 3010, Australia e-mail: smzhou@ms.unimelb.edu.au 